\title{
Is perfectionism associated with academic burnout through repetitive negative thinking?
}

\author{
David Garratt-Reed ${ }^{1}$, Joel Howell ${ }^{\text {Corresp., }}{ }^{1}$, Lana Hayes , Mark Boyes ${ }^{1}$ \\ ${ }^{1}$ School of Psychology, Curtin University of Technology, Australia \\ Corresponding Author: Joel Howell \\ Email address: joel.howell@curtin.edu.au
}

Academic burnout is prevalent among university students, although understanding of what predicts burnout is limited. This study aimed to test the direct and indirect relationship between two dimensions of perfectionism (Perfectionistic Concerns and Perfectionistic Strivings) and the three elements of Academic Burnout (Exhaustion, Inadequacy, and Cynicism) through Repetitive Negative Thinking. In a cross-sectional survey, undergraduate students ( $n=126, \mathrm{M}_{\text {age }}=23.64,79 \%$ Female) completed well-validated measures of Perfectionism, Repetitive Negative Thinking, and Academic Burnout. Perfectionistic Concerns was directly associated with all elements of burnout, as well as indirectly associated with Exhaustion and Cynicism via Repetitive Negative Thinking. Perfectionistic Strivings was directly associated with less Inadequacy and Cynicism; however, there were no indirect associations between Perfectionistic Strivings and Academic Burnout operating through Repetitive Negative Thinking. Repetitive Negative Thinking was also directly related to more burnout Exhaustion and Inadequacy, but not Cynicism. It is concluded that future research should investigate whether interventions targeting Perfectionistic Concerns and Repetitive Negative Thinking can reduce Academic Burnout in university students. 
1

2 Is perfectionism associated with academic burnout through repetitive negative thinking?

3

4

5

David Garratt-Reed ${ }^{\text {a }}$, Joel Howell ${ }^{\text {a*}}$, Lana Hayes, Mark Boyes ${ }^{\text {a }}$

6

7

8

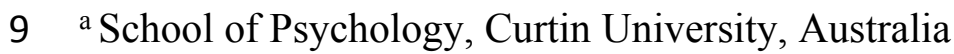

10

11

12 *Correspondence should be addressed to Joel Howell, School of Psychology, Curtin University, 13 GPO Box U1987, Perth, WA, 6845, Australia.

14 E-mail: joel.howell@curtin.edu.au 
Abstract

16

17 Academic burnout is prevalent among university students, although understanding of what

predicts burnout is limited. This study aimed to test the direct and indirect relationship between two dimensions of perfectionism (Perfectionistic Concerns and Perfectionistic Strivings) and the three elements of Academic Burnout (Exhaustion, Inadequacy, and Cynicism) through Repetitive Negative Thinking. In a cross-sectional survey, undergraduate students $\left(n=126, \mathrm{M}_{\text {age }}=23.64\right.$, 79\% Female) completed well-validated measures of Perfectionism, Repetitive Negative Thinking, and Academic Burnout. Perfectionistic Concerns was directly associated with all elements of burnout, as well as indirectly associated with Exhaustion and Cynicism via Repetitive Negative Thinking. Perfectionistic Strivings was directly associated with less Inadequacy and Cynicism; however, there were no indirect associations between Perfectionistic Strivings and Academic Burnout operating through Repetitive Negative Thinking. Repetitive Negative Thinking was also directly related to more burnout Exhaustion and Inadequacy, but not Cynicism. It is concluded that future research should investigate whether interventions targeting Perfectionistic Concerns and Repetitive Negative Thinking can reduce Academic Burnout in university students.

Individuals experiencing burnout have difficulty committing to the task at hand, feel detached and dissatisfied with their work, and are less productive (Leiter \& Maslach, 2003). Research has identified three main dimensions of burnout: Exhaustion; Cynicism; and Inadequacy. Exhaustion refers to the stress that leaves an individual feeling unable to commit to the task at hand (Maslach \& Jackson, 1981). Cynicism refers to an individual's cynical attitude toward work, which leads to negative, detached feelings towards their work (Leiter \& Maslach, 2003). Inadequacy refers to the individual feeling incompetent at work, which is often accompanied by reduced productivity and dissatisfaction with their work achievements (Leiter \& Maslach, 2003). Whilst much research has studied burnout in the workplace, recent research is exploring how these aspects of burnout present in university students, which is important given evidence that Academic Burnout is associated with poor educational outcomes (Mazurklewicz, Korenstein, Fallar, \& Ripp, 2011; Zhang, Gan, \& Cham, 2007). 
46

47

48

49

50

51

52

53

54

55

56

57

58

59

60

61

62

63

64

65

66

67

68

69

70

71

72

73

74

75

76

Similar to burnout in other contexts, Academic Burnout involves three elements:

Exhaustion due to study demands; feelings of Inadequacy as a student due to the long-term stress of striving for academic achievement; and a Cynical Attitude toward study (Merino-Tejedor, Sánchez-García, Prizmic-Kuzmica, \& Vigil-Colet, 2014; Salmela-Aro, Kiuru, Leskinen, \& Nurmi, 2009). Academic burnout is associated with various negative consequences, including poor academic outcomes, increased psychological distress, reduced life satisfaction, and sleep deprivation (e.g., May, Bauer, \& Fincham, 2015; Mazurklewicz et al., 2011; Salmela-Aro \& Upadyaya, 2014). Given that Academic Burnout is highly prevalent among university students (e.g., Kristanto, Chen, \& Thoo, 2016; Mazurklewicz et al., 2011), it is important to develop methods of reducing Academic Burnout. A vital step towards this is to identify factors associated with burnout. Perfectionism has been linked to numerous psychological symptoms (e.g., Limburg, Watson, Hagger, \& Egan, 2017) and preliminary evidence also indicates that it predicts Academic Burnout (Kljajic, Gaudreau, \& Franche, 2017). Additionally, there is emerging evidence that the relationship between perfectionism and various psychological symptoms is mediated by Repetitive Negative Thinking (Egan, Hattaway, \& Kane, 2014). Consequently, the current study will investigate whether two forms of perfectionism (Perfectionistic Concerns and Perfectionistic Strivings) predict the elements of Academic Burnout, and whether these relationships are mediated by Repetitive Negative Thinking.

Perfectionism has been implicated in the development and maintenance of a variety of psychopathologies (e.g., Limburg et al., 2017) and is also associated with Academic Burnout (Kljajic et al., 2017). Across multiple perfectionism scales, there consists of two higher order dimensions: Perfectionistic Concerns and Perfectionistic Strivings (Burgess, Frost, \& DiBartolo, 2016; Limburg et al., 2017; Stoeber \& Otto, 2006). Perfectionistic Concerns involves being overly concerned about mistakes in performance as well as doubting one's actions, and is consistently related to negative psychological outcomes (Bieling, Israeli, \& Antony, 2004; Limburg et al., 2017). Across two of the most popular perfectionism scales the Concern over Mistakes, Doubts about Actions, Parental Expectations, and Parental Criticism Subscales from the Frost Multidimensional Perfectionism Scale (FMPS; Frost, Marten, Lahart, \& Rosenblate, 1990), and Socially Prescribed Perfectionism from the Hewitt and Flett Multidimensional Perfectionism Scale (HMPS; Hewitt \& Flett, 1991) consistently load onto Perfectionistic Concerns factor (Bieling et al., 2004; Limburg et al., 2017). Perfectionistic Strivings involves the 
77 setting of high personal standards, which has been associated with both positive and negative 78 psychological outcomes (Limburg et al., 2017; Smith, et al., 2016; Stoeber \& Childs, 2010). The 79 Personal Standards and Organisation subscales of the FMPS, and the Self-Oriented

80 Perfectionism and Other-Oriented Perfectionism subscales of the HMPS consistently load onto 81 the Perfectionistic Strivings factor (Bieling et al., 2004; Limburg et al., 2017).

82

83

84 85

The relationship between perfectionism and burnout has been examined in various nonacademic contexts, with Perfectionistic Concerns reliably associated with higher burnout, and aspects of burnout (Exhaustion, Inadequacy, and Cynicism), in many samples, including teachers and junior athletes (e.g., Hill, 2013, Stoeber \& Rennert, 2008). In contrast, Perfectionistic Strivings has been associated with lower levels of burnout, although the relationship is somewhat inconsistent across the various aspects of burnout (Hill, 2013; Stoeber \& Rennert, 2008).

The few studies that have directly examined the relationship between perfectionism and Academic Burnout have reported broadly consistent findings. Using the Chinese translation of the Frost Multidimensional Perfectionism Scale, Zhang and colleagues (2007) demonstrated that Perfectionistic Concerns (measured by Concern over Mistakes, Doubts about Actions, and Parental Expectations subscales) and Perfectionistic Strivings (measured by Personal Standards and Organisation subscales) predicted aspects of Academic Burnout in a sample of 482 Chinese university students. Specifically higher levels of Perfectionistic Concerns predicted more Exhaustion and Cynicism, and less engagement with university. Higher levels of Perfectionistic Strivings predicted lower levels of Exhaustion and Cynicism, and more engagement. These results suggest that individuals with higher levels of Perfectionistic Concerns are more likely to experience higher levels of Academic Burnout, whilst individuals with higher levels of Perfectionistic Strivings are likely to experience lower Academic Burnout and higher study efficacy.

Kljajic and colleagues (2017) categorised students as either pure socially prescribed perfectionists, pure self-oriented perfectionists, mixed perfectionists (involving high levels of socially prescribed and self-oriented perfectionism), or non-perfectionistic. Socially prescribed perfectionists (related to Perfectionistic Concerns) were more likely to experience Academic Burnout, as measured separately by Exhaustion, Cynicism, and study efficacy, than nonperfectionists or mixed perfectionists. Moreover, self-oriented perfectionists (related to Perfectionistic Strivings) were less likely to experience Academic Burnout than non- 
108 perfectionists and mixed perfectionists. Perfectionism also predicts Academic Burnout among 109 high school students (Shih, 2012). Despite the emerging evidence, few studies have considered 110 the possible role of variables that may mediate the association between perfectionism and

111 Academic Burnout. Repetitive Negative Thinking is one potential candidate that warrants 112 investigation.

113 Repetitive Negative Thinking is an unhelpful continual thought process about past and/or

114 future negative situations, leading to negative emotional states (McLaughlin \& Nolen-Hoeksema, 115 2011). It appears to be a risk factor for the development of numerous types of psychopathology 116 (e.g., McEvoy, Watson, Watkins, \& Nathan, 2013). Interestingly, Repetitive Negative Thinking 117 mediates the relationship between perfectionism and various psychological difficulties, including 118 depression (Flett, Coulter, Hewitt, \& Nepon, 2011), post-traumatic stress disorder (Egan et al., 119 2014), and psychological distress (Macedo et al., 2015; O'Connor, O'Connor, \& Marshall, 120 2007). Macedo and colleagues (2015) found that Repetitive Negative Thinking partially 121 mediated the positive relationship between Perfectionistic Concerns and aspects of psychological 122 distress (anxiety, depression, anger-hostility, fatigue, and vigor). Interestingly, Repetitive 123 Negative Thinking fully mediated the relationship between Perfectionistic Strivings and 124 depression and fatigue, meaning that Perfectionistic Strivings only predicted higher distress 125 through its relationship with Repetitive Negative Thinking. However, it should be noted that 126 Perfectionistic Strivings did not predict the other aspects of distress in this study. Given the 127 associations between Repetitive Negative Thinking and both perfectionism and other 128 psychological outcomes, it is plausible that Repetitive Negative Thinking may mediate 129 associations between perfectionism and Academic Burnout.

130 The current study aimed to 1) replicate associations between perfectionism 131 (Perfectionistic Concerns and Perfectionistic Strivings) and Academic Burnout (Exhaustion, 132 Inadequacy, and Cynicism) and 2) investigate whether Repetitive Negative Thinking mediates 133 the relationships between perfectionism and the elements of Academic Burnout. The 134 hypothesised model is summarised in Figure 1. The model tested the direct pathways between 135 perfectionism and burnout, as well as the indirect relationships operating via Repetitive Negative 136 Thinking.

\section{Method}




\section{Participants}

140

141

142

143

144

145

146

147

148

149

150

151

152

153

154

155

156

157

158

159

160

161

162

163

164

165

166

167

168

169

The sample initially consisted of 215 Australian university students aged 18 and over.

However, 89 individuals simply opened and closed the survey without indicating consent or responding to any items, leaving a final sample of 126. The final sample (100 females, 25 males, 1 transgender) ranged in age from 18-69 $(\mathrm{M}=23.64, \mathrm{SD}=7.86)$, years at university from 1-8 years $(\mathrm{M}=3.07, \mathrm{SD}=1.56)$, and studying predominantly full-time (104 full time, 22 part time). Of the 126 participants, 57 were recruited from a research participation pool from the School of Psychology at Curtin University and received course credit for participation, while 69 were recruited from other courses via social media, though specific courses were not recorded. There were no significant differences between the mean scores of these two sample groups on any measure used in the study.

\section{Measures}

Demographics. Demographic questions measured age, gender, study mode (i.e. full-time or part-time), and number of years completed at university.

Frost Multidimensional Perfectionism Scale - Brief (FMPS-Brief; Burgess et al., 2016).

The FMPS-Brief (Burgess et al., 2016) is an 8-item scale measuring two aspects of perfectionism: Perfectionistic Concerns (four items, e.g., If I fail at university, I am a failure as a person) and Perfectionistic Strivings (four items, e.g., I have extremely high goals). Items are rated on a 5-point Likert scale ranging from strongly disagree (1) to strongly agree (5). Scores are summed (ranging between 4 and 20), with higher scores indicative of higher levels of Perfectionistic Concerns or Strivings. The Perfectionistic Concerns subscale is strongly, positively correlated with measures of depression, anxiety, hoarding, and worry, indicating good convergent validity (Burgess et al., 2016; Limburg et al., 2017). The Perfectionistic Concerns and Strivings subscales have demonstrated good internal consistency in community samples previously ( $\alpha=.83$ and $\alpha=.81$, respectively Burgess et al., 2016), as well as in the present study ( $\alpha=.78$ and $\alpha=.88$, respectively).

Repetitive Negative Thinking - 10 (RNT-10; McEvoy, Mahoney, \& Moulds, 2010). The RNT-10 was adapted from the Repetitive Negative Thinking Scale (McEvoy et al., 2010). It contains 10 items assessing engagement with Repetitive Negative Thinking (e.g., Once you start thinking about the situation, you can't stop). Items are rated on a 5-point Likert scale ranging from not true at all (1) to very true (5). Responses from all 10 items are summed (ranging 
170 between 10 and 50), with higher scores indicate higher levels of engagement with Repetitive

171 Negative Thinking. The RNT-10 is positively correlated with measures of neuroticism,

172 depression, social anxiety, and worry, indicating convergent validity (Mahoney, McEvoy, \&

173 Moulds, 2012). The RNT-10 demonstrated scale reliability in initial development ( $\alpha=.89$,

174 average inter-item correlation $=.44$; McEvoy et al., 2010) and subsequent research $(\alpha=.91$,

175 average inter-item correlation $=.49$; Mahoney et al., 2012). In the present study the internal

176 consistency of the RNT-10 was high $(\alpha=.94)$.

177 School Burnout Inventory (SBI; Salmela-Aro \& Näätänen, 2005). The SBI (Salmela-Aro

178 \& Näätänen, 2005) is a 9-item questionnaire with 3 subscales: Exhaustion at School (four items,

179 e.g., I feel overwhelmed by my university work), Cynicism Toward Meaning of School (three

180 items, e.g., I feel that I am losing interest in my schoolwork), and Sense of Inadequacy at School

181 (two items, e.g., I often have feelings of inadequacy in my university work). Items are rated on a

182 6-point Likert-type scale ranging from completely disagree (1) to strongly agree (6), with

183 subscale scores calculated by summing the respective items. Scores range between 4 and 24 for

184 the Exhaustion subscale, 3 and 18 for Cynicism subscale, and 2 and 12 for the Inadequacy

185 subscale. Higher scores reflect higher levels of each construct (Salmela-Aro \& Näätänen, 2005).

186 All items had references to 'school' changed to 'university' to reflect the university context in

187 this study. The SBI is correlated with academic achievement, and measures of depression and

188 engagement, demonstrating concurrent validity (Salmela-Aro et al., 2009). The SBI

189 demonstrated good internal consistency for subscales ( $\alpha=.67-.80$; Salmela-Aro et al., 2009). In

190 the present study, the Exhaustion and Cynicism subscales demonstrated good internal

191 consistency ( $\alpha=.81$ and .85 , respectively), but the internal consistency of the inadequacy

192 subscale was low $(\alpha=.54)$.

193 Procedure

194

195

196

(RDHS-91-16). Participants accessed the anonymous questionnaire online, whereby they viewed a participant information document and provided informed consent before completing the

197 questionnaire, hosted through Qualtrics. Following questionnaire completion, participants were

198 presented with a debriefing document that outlined the purpose of the research and informed

199 them of where to find more information. Participants who required research participation for their course were credited participation points for completing the survey. 


\section{Data analysis}

202 The hypothesised model was tested using path analysis in Mplus. The significance values

203 for both direct and indirect pathways were estimated with a 95\% confidence interval using a

204 bootstrapping procedure based on 1000 draws from the data. Modification Indices (MIs $>20, \mathrm{Hu}$

$205 \&$ Bentler, 1999) were examined and theoretically defensible paths were freed. Goodness-of-fit

206 was assessed using the chi-square statistic and degrees of freedom (Chi-square/df), Comparative

207 Fit Index (CFI; values should be $\geq 0.95$ ), Root Mean Square Error of Approximation (RMSEA;

208 values should be $\leq 0.06$ ), Tucker-Lewis Index (TLI; values should be $\geq 0.95$ ), and Standardised

209 Root Mean Square Residual (SRMR; values should be $\leq 0.08$, Hu \& Bentler, 1999). Although

210 there was no significant correlation between age and gender and perfectionism, Repetitive

211 Negative Thinking, and Academic Burnout, the model was run with and without the control

212 variables of age and gender and the pattern of significant results did not change. Therefore

213 results from the most parsimonious models without control variables are reported. The

214 correlation between Perfectionistic Concerns and Strivings were controlled for, as were the

215 correlations between burnout Exhaustion, Inadequacy, and Cynicism.

216

Results

217 Descriptive statistics and correlations between all variables of interest are summarised in Table

218 1. Correlations were generally in the expected directions and were small to moderate in

219 magnitude. The present sample demonstrated scores that were consistent with the non-clinical

220 samples in other studies for both perfectionism scales (Burgess et al., 2016), and Repetitive

221 Negative Thinking (McEvoy et al., 2010). Scores were slightly higher for the School Burnout

222 Inventory relative to the high school sample reported by Salmela-Aro and Näätänen (2005).

223

224

225

226

227

228

229

230

231

232

[Insert Table 1 about here]

\section{Path Analysis Models}

Initial Model. A test of the full model indicated a just-identified model, from which fit statistics were not produced (Supplementary Figure 1). In this model there were statistically significant direct positive effects of Perfectionistic Concerns on Repetitive Negative Thinking, Burnout Exhaustion, Burnout Inadequacy, and Burnout Cynicism. There was also a statistically significant direct effect of Repetitive Negative Thinking on Burnout Exhaustion and Burnout Inadequacy. Additionally, there were statistically significant direct positive effects of 
233 Perfectionistic Strivings on Burnout Inadequacy and Burnout Cynicism. There was no direct

234 relationship between Perfectionistic Strivings and Repetitive Negative Thinking or Burnout

235 Exhaustion. There was also no direct relationship between Repetitive Negative Thinking and

236 Burnout Cynicism.

237 Model 2. A second model was run, without the non-significant pathways to test the most

238 parsimonious model. The path analysis revealed good model fit to the data $\chi^{2} / \mathrm{df}=1.07, \mathrm{CFI}=$ $239.999, \mathrm{TLI}=.995, \mathrm{RMSEA}=.024(90 \% \mathrm{CI}=.000 ; .154), \mathrm{SRMR}=.030($ see Figure 2$)$.

Direct pathways. There were statistically significant direct positive effects of

241 Perfectionistic Concerns on Repetitive Negative Thinking, Burnout Exhaustion $(p<.001)$,

242 Burnout Inadequacy $(p<.001)$, and Burnout Cynicism $(p<.001)$. There was also a statistically

243 significant direct effect of Repetitive Negative Thinking on Burnout Exhaustion $(p=.001)$, and

244 Burnout Inadequacy $(p=.041)$. Additionally, there were statistically significant direct positive

245 effects of Perfectionistic Strivings on Burnout Inadequacy $(p<.001)$, and Burnout Cynicism $(p=$ $246.017)$.

247

Indirect pathways. There were significant indirect relationships between Perfectionistic

248 Concerns and Burnout Exhaustion $(\beta=.121, p=.003, \mathrm{SE}=0.041,95 \% \mathrm{CI}=.055-.190)$. No

249 other indirect pathways were observed. See Figure 2 for the final path analysis model with

250 standardised beta, standard error, and 95\% confidence intervals for significant pathways.

251

[Insert figure 2 about here]

252

253

\section{Discussion}

The aim of the study was to investigate the relationship between perfectionism,

254 Repetitive Negative Thinking, and Academic Burnout. Consistent with previous research (e.g., 255 Kljajic et al., 2017), Perfectionistic Concerns was independently associated with all three 256 elements of Academic Burnout among university students. These findings suggest that

257 Perfectionistic Concerns is an important predictor of Academic Burnout among university 258 students and represents a viable target for interventions aimed at reducing burnout in this 259 population. Previous research has demonstrated that cognitive-behavioural programs are 260 effective in reducing problematic Perfectionistic Concerns and that interventions specifically 261 targeting perfectionism can reduce various psychological symptoms, including eating disorder 262 symptoms, generalised anxiety, and depression (e.g., Rozental et al., 2017; Shafran et al., 2017; 263 also see Egan, Wade, \& Shafran, 2011 for a review). Future studies should investigate whether 
264 such interventions targeting Perfectionistic Concerns are similarly effective in reducing

265 Academic Burnout in university students. Not only would the results of such studies have 266 considerable practical utility, they would also further understanding of the causal nature of the 267 relationship between Perfectionistic Concerns and Academic Burnout.

268 Additionally, Repetitive Negative Thinking partially mediated the association between 269 Perfectionistic Concerns and Exhaustion. The indirect relationship between Perfectionistic 270 Concerns and Exhaustion via Repetitive Negative Thinking is broadly consistent with recent 271 research demonstrating that the relationship between perfectionism and various psychological 272 constructs, including burnout, is partially mediated by other variables such as motivation, self273 esteem, and coping styles (e.g., Chang, Lee, Byeon, \& Lee, 2015; Luo, Wang, Zhang, Chen, \& 274 Quan, 2016; Macedo et al., 2015). It is also consistent with findings that Repetitive Negative 275 Thinking is a risk factor for multiple psychological symptoms (McEvoy et al., 2013) and that it 276 mediates the relationship between perfectionism and various psychological outcomes (e.g., Flett 277 et al., 2011).

$278 \quad$ Furthermore, Repetitive Negative Thinking was also directly associated with burnout 279 Exhaustion and burnout Inadequacy. This highlights the importance of investigating the efficacy 280 of therapeutic programs targeting Repetitive Negative Thinking for students who have high 281 levels of Academic Burnout (particularly that characterised by Exhaustion) and high levels of 282 Perfectionistic Concerns. Repetitive Negative Thinking can be reduced through targeted 283 interventions (Watkins 2018), or through metacognitive therapy, and this is associated with 284 improvements in several measures of psychological distress (Johnson, Hoffart, Nordahl, \& 285 Wampold, 2017; McEvoy, Erceg-Hurn, Anderson, Campbell, \& Nathan, 2015) or through. The 286 importance of Repetitive Negative Thinking as a treatment for burnout would critically depend 287 upon whether any modification of a student's Repetitive Negative Thinking is also associated 288 with changes in their experiences of burnout. Future research should investigate the efficacy of 289 such programs in reducing burnout Exhaustion in university students. It is also important to 290 recognise the potential overlap between Repetitive Negative Thinking and depressive disorders 291 (Nolen-Hoeksema, 2000) and anxiety disorders (McEvoy et al., 2013). Although Repetitive 292 Negative Thinking can be considered a transdiagnostic process (Harvey, Watkins, Mensell, \& 293 Shafran, 2004) and is related to burnout, it is unclear whether Repetitive Negative Thinking 294 better explains burnout relative to specific symptoms of depression and anxiety. As such, it 
295 would be informative for future research to measure all of these constructs and investigate 296 whether they are independently associated with burnout.

297 Perfectionistic Strivings was associated with lower levels of Academic Burnout, 298 specifically Inadequacy and Cynicism. This is consistent with previous findings linking 299 constructs closely related to Perfectionistic Strivings (adaptive perfectionism and self-oriented 300 perfectionism) with lower burnout (Klajic et al., 2017; Zhang et al., 2007). This finding is also 301 consistent with the notion that Perfectionistic Strivings can be a beneficial form of perfectionism 302 (e.g., Stoeber \& Childs, 2010). However, given evidence that Perfectionistic Strivings might 303 predict negative psychological outcomes in longitudinal studies (Smith et al., 2016), this 304 interpretation requires caution. Finally, Perfectionistic Strivings was not indirectly associated 305 with any aspect of Academic Burnout via Repetitive Negative Thinking. Somewhat surprisingly, 306 Perfectionistic Strivings was not associated with Repetitive Negative Thinking. This is 307 inconsistent with the results of Macedo and colleagues (2015), who demonstrated that 308 Perfectionistic Strivings predicts depression and fatigue through Repetitive Negative Thinking. 309 Consequently, the relationship between Perfectionistic Strivings and Repetitive Negative 310 Thinking requires further investigation.

Based on the current findings, it is plausible that reducing a students' concern regarding 312 mistakes (e.g., Perfectionistic Concerns), may help to reduce Academic Burnout. In many 313 interventions for perfectionism, it is not about reducing an individual's own standards, but rather 314 about promoting a healthy striving for excellence without the individual then basing their self315 worth on the striving for or achievement of their high goals (Egan, van Noort, et al., 2014; 316 Handley, Egan, Kane, \& Rees, 2015; Egan, Wade et al., 2014). Nehmy and Wade (2015) have 317 also tested an intervention for perfectionism within schools that demonstrated improvements in 318 perfectionism, self-criticism, and negative affect at 6-months post intervention, with 319 improvements in perfectionism and self-criticism maintained at 12-months post intervention. As 320 Nehmy and Wade demonstrated some preventative effects through their school based 321 intervention, it may also be of use to evaluate whether such a program also provides some 322 preventative effects for academic burnout.

323 The findings of the present study should be interpreted within the context of the 324 limitations. First, the cross-sectional nature of the data precludes any conclusions regarding the 325 temporal order of the associations. Longitudinal research is clearly needed to address this. 
326 Additionally, longitudinal data is required to address the possibility that the relationship between

327 Perfectionistic Concerns, Repetitive Negative Thinking, and Academic Burnout varies

328 depending upon the stage of a student's academic career. It is plausible that the relationship is

329 more pronounced at various time points in semester (e.g., before assignments or exams are due

330 versus after assessment deadlines) and future research should therefore further examine these

331 factors in future academic burnout research. Second, the sample was one of convenience and this

332 may limit the generalisability of the findings to the broader student population. Third, the

333 reliability of the Inadequacy subscale was low. Further psychometric assessment of this scale in

334 university samples is needed and findings in the current study related to burnout Inadequacy

335 should be interpreted with caution. Finally, Repetitive Negative Thinking is a theoretically

336 plausible mediator between perfectionism and Academic Burnout because it is a pathway

337 between perfectionism and depression (Flett, Coulter, Hewitt, \& Nepon, 2011), post-traumatic

338 stress disorder (Egan et al., 2014), and psychological distress (Macedo et al., 2015). However,

339 Repetitive Negative Thinking is only one of many potential factors that might mediate the

340 relationship between perfectionism and Academic Burnout. Future research should examine

341 other potential mediators or moderators. For example, one potential possibility is academic

342 procrastination, which is positively related to Perfectionistic Concerns and negatively related to

343 wellbeing (Jadidi, Mohammadkhani, \& Tajrishi, 2001; Steel, 2007). Additionally, individual

344 differences in factors such as imagery, emotion regulation, and coping can exert a strong

345 influence on psychological outcomes (Holmes, Geddes, Colom, \& Goodwin, 2008). These

346 variables may be important to consider in the context of Academic Burnout in order to best

347 improve student outcomes.

\section{Conclusions}

349 Bearing the limitations in mind, the current study demonstrates that higher levels of

350 Perfectionistic Concerns are associated with greater experiences of Academic Burnout, both

351 directly and indirectly through increased Repetitive Negative Thinking in the case of burnout

352 Exhaustion. Repetitive Negative Thinking was also directly associated with burnout Exhaustion

353 and burnout Inadequacy. In contrast, higher levels of Perfectionistic Strivings are associated with

354 less Academic Burnout. Given that there are treatment programs which can effectively reduce

355 both Perfectionistic Concerns and Repetitive Negative Thinking (McEvoy et al., 2015; Rozental 
356 et al., 2017), future research should focus on understanding the extent to which these programs 357 can alleviate Academic Burnout in university students. 
359 Bieling, P. J., Israeli, A. L., \& Antony, M. A. (2004). Is perfectionism good, bad, or both?

360

361 Examining models of the perfectionism construct. Personality and Individual Differences,

362 Burgess, M. B., Frost, R. O., \& DiBartolo, P. M. (2016). Development and validation of the Frost 363 Multidimensional Scale-Brief. Journal of Psychological Assessment, 34, 1-14.

365 Chang, E., Lee, A., Byeon, E., \& Lee, S. M. (2015). Role of motivation in the relation between 366 perfectionism and academic burnout in Korean students. Personality and Individual

368 Egan, S. J., Hattaway, M., \& Kane, R. T. (2014). The relationship between perfectionism and 369

370 rumination in post traumatic stress disorder. Behavioural and Cognitive Psychotherapy, 42,

371 Egan, S. J., van Noort, E., Chee, A., Kane, R. T., Hoiles, K. J., Shafran, R., \& Wade, T. D. (2014).

372

373

374 A randomised controlled trial of face to face versus pure online self-help cognitive behavioural treatment for perfectionism. Behaviour Research and Therapy, 63C, 107-113.

375 Egan, S. J., Wade, T. D., \& Shafran, R. (2011). Perfectionism as a transdiagnostic process: A $376 \quad$ clinical review. Clinical Psychology Review, 31, 203-212. doi:10.1016/j.cpr.2010.04.009

377 Frost, R. O., Marten, P., Lahart, C., \& Rosenblate, R. (1990). The dimensions of

378 perfectionism. Cognitive Therapy and Research, 14(5), 449-468. doi:

379 10.1007/BF01172967

380 Flett, G. L., Coulter, L. M., Hewitt, P. L., \& Nepon, T. (2011). Perfectionism, rumination, worry 381

382 and depressive symptoms in early adolescents. Canadian Journal of School Psychology, 26, 159-176. doi:10.1177/0829573511422039

383 Hayes, A. F. (2013). Introduction to Mediation, Moderation, and Conditional Process Analysis: A 384 Regression-Based Approach. Guilford, NY: Guilford.

385 Harvey, A., Watkins, E., Mansell, W., \& Shafran, R. (2004). Cognitive behavioural processes 386 across psychological disorders: A transdiagnostic approach to research and treatment. Oxford, UK: Oxford University Press.

388 Handley, A. K., Egan, S. J., Kane, R. T., \& Rees, C. S. (2015). A randomised controlled trial of 
389

390

group cognitive behavioural therapy for perfectionism. Behaviour Research and Therapy, 68, 37-47. doi:10.1016/j.brat.2015.02.006

391 Hewitt, P. L., \& Flett, G. L. (1991). Perfectionism in the self and social contexts:

392 Conceptualization, assessment and association with psychopathology. Journal of

393 Personality and Social Psychology, 60(3), 456-470. doi:10.1037/0022-3514.60.3.456

394 Hill, A. P. (2013). Perfectionism and burnout in junior soccer players: A test of the 2x2 model of 395 dispositional perfectionism. Journal of Sport and Exercise Physiology, 35, 18-29.

396 doi:10.1123/jsep.35.1.18

397 Holmes, E. A., Geddes, J. R., Colom, F., \& Goodwin, G. M. (2008). Mental imagery as an 398 emotional amplifier: Application to bipolar disorder. Behaviour Research and Therapy, 399 46(12), 1251-1258. doi:10.1016/j.brat.2008.09.005

$400 \mathrm{Hu}$, L. T., \& Bentler, P. M. (1999). Cutoff criteria for fit indexes in covariance structure analysis:

401 Conventional criteria versus new alternatives. Structural Equation Modeling: A

402 Multidisciplinary Journal, 6, 1-55. doi:10.1080/10705519909540118

403 Jadidi, F., Mohammadkhani, S., \& Tajrishi, K. Z. (2011). Perfectionism and academic 404 procrastination. Procedia-Social and Behavioral Sciences, 30, 534-537.

405 doi:10.1016/j.sbspro.2011.10.104

406 Johnson, S. U., Hoffart, A., Nordahl, H. M., \& Wampold, B. E. (2017). Metacognitive therapy 407 versus disorder-specific CBT for comorbid anxiety disorders: A randomized controlled 408 trial. Journal of Anxiety Disorders, 50, 103-112. doi:10.1016/j.janxdis.2017.06.004

409 Kljajic, K., Gaudreau, P., \& Franche, V. (2017). An investigation of the 2x2 model of 410 perfectionism with burnout, engagement, self-regulation, and academic burnout. Learning 411 and Individual Differences, 57, 103-113. doi:10.1016/j.lindif.2017.06.004

412 Kristanto, T., Chen, W. S., \& Thoo, Y. Y. (2016). Academic burnout and eating disorder among 413 students in Monash University Malaysia. Eating Behaviors, 22, 96-100.

414 doi:10.1016/j.eatbeh.2016.03.029

415 Leiter, M. P., \& Maslach, C. (2003). Areas of worklife: A structured approach to organizational 416 predictors of job burnout. Research in Occupational Stress and Well Being, 3, 91-134. 417 doi:10.1016/S1479-3555(03)03003-8 
418 Limburg, K., Watson, H. J., Hagger, M. S., \& Egan, S. J. (2017). The relationship between

419

420

421 Luo, Y., Wang, Z., Zhang, H., Chen, A., \& Quan, S. (2016). The effect of perfectionism on school perfectionism and psychopathology: A meta-analysis. Journal of Clinical Psychology, 73,

422

423 burnout among adolescence: The mediator of self-esteem and coping style. Personality and 424 Macedo, A., Soares, M. J., Amaral, A. P., Nogueira, V., Madeira, N., Roque, C., .. Pereira, A. T. 425

426

427

428 Mahoney, A. E., McEvoy, P. M., \& Moulds, M. L. (2012). Psychometric properties of the 429

430

431 Maslach, C., \& Jackson, S. E. (1981). The measurement of experienced burnout. Journal of 432 Organizational Behavior, 2, 99-113. doi:10.1002/job.4030020205

433 May, R. W., Bauer, K. N., \& Fincham, F. D. (2015). School burnout: Diminished academic 434 435 and cognitive performance. Learning and Individual Differences, 42, 126-131.

doi:10.1016/j.lindif.2015.07.015

436 Mazurklewicz, R., Korenstein, D., Fallar, R., \& Ripp, J. (2011). The prevalence and correlations 437 of medical student burnout in the pre-clinical years: A cross-sectional study. Psychology, $438 \quad$ Health and Medicine, 17, 188-195. doi:10.1080/13548506.2011.597770

439 McEvoy, P. M., Erceg-Hurn, D. M., Anderson, R. A., Campbell, B. N., \& Nathan, P. R. (2015). 440 Mechanisms of change during group metacognitive therapy for repetitive negative thinking 441 in primary and non-primary generalized anxiety disorder. Journal of Anxiety Disorders, 35, 442 19-26. doi:10.1016/j.janxdis.2015.07.003

443 McEvoy, P. M., Mahoney, A. E., \& Moulds, M. L. (2010). Are worry, rumination, and post-event $444 \quad$ processing one and the same?: Development of the Repetitive Thinking Questionnaire. 445 Journal of Anxiety Disorders, 24, 509-519. doi:10.1016/j.janxdis.2010.03.008 446 McEvoy, P. M., Watson, H., Watkins, E. R., \& Nathan, P. (2013). The relationship between 447 worry, rumination, and comorbidity: Evidence for repetitive negative thinking as a 
448 transdiagnostic construct. Journal of Affective Disorders, 151, 313-320.

449 doi:10.1016/j.jad.2013.06.014

450 McLaughlin, K. A., \& Nolen-Hoeksema, S. (2011). Rumination as a transdiagnostic factor in 451 depression and anxiety. Behaviour Research and Therapy, 49, 186-193.

452 doi:10.1016/j.brat.2010.12.006

453 Merino-Tejedor, E., Sánchez-García, J. C., Prizmic-Kuzmica, A. J., \& Vigil-Colet, A. (2014).

454 Adaptation and psychometric properties of the SBI-U scale for academic burnout in

455 university students. Anales de Psicología/Annals of Psychology, 31, 290-297.

456 doi:10.6018/analesps.31.1.168581

457 Muthén, L. K., \& Muthén, B. O. (1998). Mplus user’s guide (7th ed.). Los Angeles CA: Muthén \& $458 \quad$ Muthén.

459 Nehmy, T. J., \& Wade, T. D. (2015). Reducing the onset of negative affect in adolescents:

460 Evaluation of a perfectionism program in a universal prevention setting. Behaviour

$461 \quad$ Research and Therapy, 67, 55-63. doi:10.1016/j.brat.2015.02.007

462 Nolen-Hoeksema, S. (2000). The role of rumination in depressive disorders and mixed 463 anxiety/depressive symptoms. Journal of Abnormal Psychology, 109, 504-511.

464 O’Connor, D. B., O’Connor, R. C., \& Marshall, R. (2007). Perfectionism and psychological 465 distress: Evidence of the mediating effects of rumination. European Journal of Personality, 466 21, 429-452. doi:10.1002/per.616

467 Rozental, A., Shafran, R., Wade, T., Egan, S., Nordgren, L. B., Carlbring, P., ... Andersson, G. 468 (2017). A randomized controlled trial of Internet-Based Cognitive Behavior Therapy for 469 perfectionism including an investigation of outcome predictors. Behaviour Research and $470 \quad$ Therapy, 95, 79-86. doi:10/1016/j.brat.2017.05.015

471 Salmela-Aro, K., Kiuru, N., Leskinen, E., \& Nurmi, J. E. (2009). School Burnout Inventory (SBI) 472 reliability and validity. European Journal of Psychological Assessment, 25, 48-57. 473 doi:10.1027/1015-5759.25.1.48

474 Salmela-Aro, K., \& Näätänen, P. (2005). Method of assessing adolescents'school burnout. 475 Helsinki, Finland: Edita.

476 Salmela-Aro, K., \& Upadyaya, K. (2014). School burnout and engagement in the context of 477 demands-resources model. British Journal of Educational Psychology, 84, 137-151. 478 doi:10.1111/bjep. 12018 
479 Shafran, R., Wade, T. D., Egan, S. J., Kothari, R., Allcott-Watson, H., Carlbring, P., . .

480 Andersson, G. (2017). Is the devil in the detail? A randomised controlled trial of guided

481 internet-based CBT for perfectionism. Behaviour Research and Therapy, 95, 99-106.

482 doi:10.1016/j.brat.2017.05.014

483 Shih, S-S. (2012). An examination of academic burnout versus work engagement among

484 Taiwanese adolescents. The Journal of Educational Research, 105, 286-298.

485 doi:10.1080/00220671.2011.629695

486 Smith, M. M., Sherry, S. B., Rnic, K., Saklofske, D. H., Enns, M., \& Gralnick, T. (2016). Are

487 perfectionism dimensions vulnerability factors for depressive symptoms after controlling

488 for neuroticism? A meta-analysis of 10 longitudinal studies. Europea Journal of

489 Personality, 30, 201-212. doi:10.1002/per.2053

490 Steel, P. (2007). The nature of procrastination: A meta-analytic and theoretical review of

491 quintessential self-regulatory failure. Psychological Bulletin, 133(1), 65-94.

492 doi:10.1037/0033-2909.133.1.65

493 Stoeber, J., \& Childs, J. H. (2010). The assessment of self-oriented and socially prescribed

494 perfectionism: Subscales make a difference. Journal of Personality Assessment, 92, 577-

495 585. doi:10.1080/00223891.2010.513306

496 Stoeber, J., \& Otto, K. (2006). Positive conceptions of perfectionism: Approaches, evidence,

497 challenges. Personality and Social Psychology Review, 10, 295-319.

498 doi:10.1207/s15327957pspr1004_2

499 Stoeber, J., \& Rennert, D. (2008). Perfectionism in school teachers: Relations with stress

500 appraisals, coping styles, and burnout. Anxiety, Stress, and Coping, 21, 37-53.

501 doi:10.1080/10615800701742461

502 Watkins, E. R. (2018). Rumination-focused cognitive-behavioral therapy for depression. Guilford 503 Publications.

504 Zhang, Y., Gan, T., \& Cham, H. (2007). Perfectionism, academic burnout and engagement among

505 Chinese college students: A structural equation modeling analysis. Personality and

506 Individual Differences, 43, 1529-1540. doi:10.1016/j.paid.2007 .04.01 


\section{Figure 1 (on next page)}

\section{Hypothesised model}

Complete hypothesised model of direct pathways between perfectionism, burnout, and indirect effects through repetitive negative thinking 


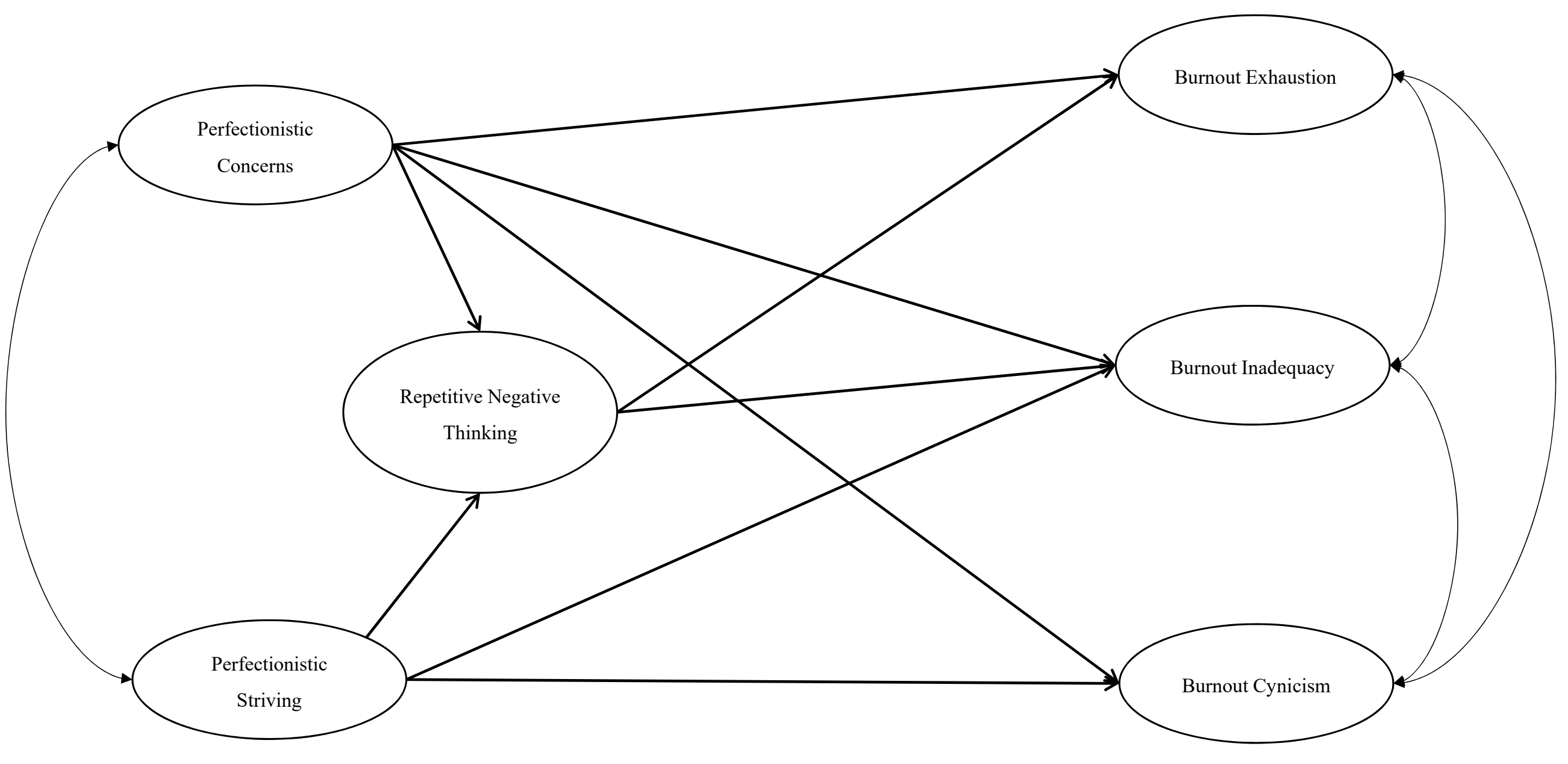




\section{Figure 2 (on next page)}

Final tested model

Final tested model with only significant pathways between perfectionism, burnout, and repetitive negative thinking considered. Only significant pathways coefficients represented. All coefficients are standardized with 95\% confidence intervals in brackets 


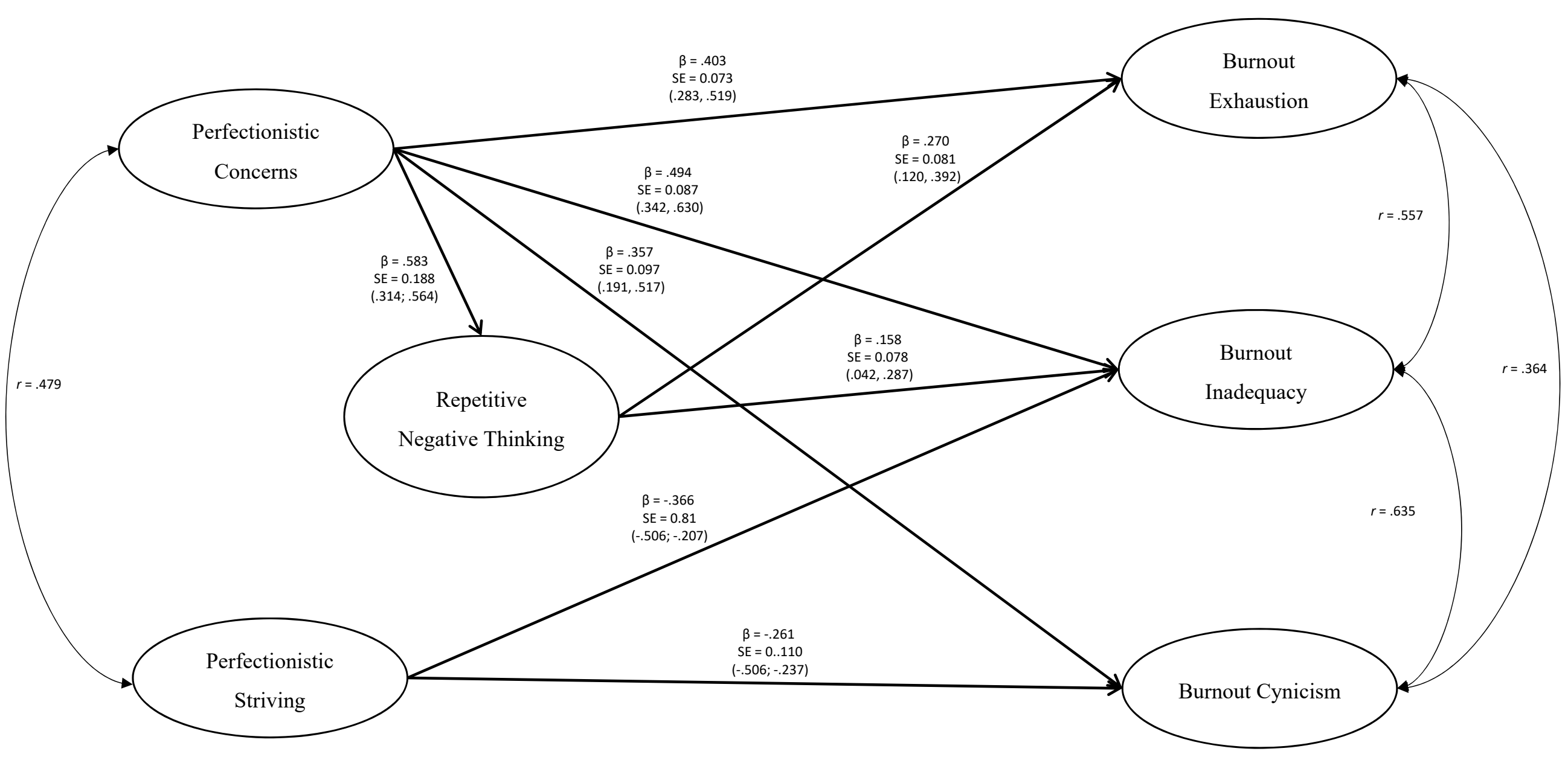




\section{Table $\mathbf{1}$ (on next page)}

Descriptive Statistics and Correlation matrix of Perfectionism, Repetitive Negative Thinking, and Burnout Measures ( $N=126)$ 
1 Table 1. Descriptive Statistics and Correlation matrix of Perfectionism, Repetitive Negative Thinking, and Burnout Measures (N=126)

\begin{tabular}{|c|c|c|c|c|c|c|c|c|c|c|}
\hline Measure & Mean(SD) & $\alpha$ & 1 & 2 & 3 & 4 & 5 & 6 & 7 & 8 \\
\hline 1. Perfectionistic Concerns & $11.25(3.61)$ & .78 & 1 & $.48^{* *}$ & $.45^{* *}$ & $.52^{* *}$ & $.23^{* *}$ & $.38^{* *}$ & .01 & .16 \\
\hline 2. Perfectionistic Strivings & $13.68(3.76)$ & .88 & & 1 & $.25^{* *}$ & $.22^{* *}$ & -.10 & -.11 & .12 & -.01 \\
\hline 3. Repetitive Negative Thinking & $34.94(8.89)$ & .94 & & & 1 & $.48^{* *}$ & $.22^{*}$ & $.35^{* *}$ & -.11 & .06 \\
\hline 4. Burnout Exhaustion & $14.98(4.60)$ & .81 & & & & 1 & $.40^{* *}$ & $.58^{* *}$ & -.01 & .17 \\
\hline 5. Burnout Cynicism & $10.56(4.11)$ & .85 & & & & & 1 & $.65^{* *}$ & -.06 & .11 \\
\hline 6. Burnout Inadequacy & $7.54(2.45)$ & .54 & & & & & & 1 & -.01 & .12 \\
\hline 7. Age & $23.64(7.86)$ & - & & & & & & & 1 & -.01 \\
\hline 8. Gender & - & - & & & & & & & & 1 \\
\hline
\end{tabular}

$2 * \mathrm{p}<.05 ; * * \mathrm{p}<.01$

3

4 